\title{
LÉGISLATION
}

\section{La Concession hydraulique, donnée en conformité de la Loi du 16 octobre 1919, est une concession de travaux publics}

\author{
par Paul Bougault, avocat à la Cour d'appel de Lyon.
}

\begin{abstract}
D'un arrêt qui paraît résoudre une simple difficulté de procédure (question du tribunal compétent pour apprécier les dommages apportés à une propriété privée par un abatage intempestif des arbres), notre collaborateur Paul Bougault déduit des règles précises : au point de vue des principes juridiques la définition exacte de la concession dite "hydraulique " est importante; elle a des répercussions nombreuses sur les décisions à prendre dans la pratique (1).
\end{abstract}

Faits ayant donné lieu au procès. - La Société Hydroélectrique et Métallurgique du Palais nantie d'un décret en date du 28 juin 1923 (voir Bulletin de la Chambre Syndicale des Forces Hydrauliques, année 1923, No 440), pour la constitution d'une chute sur le Taurion (puissance normale disponible 5.500 kilowatts environ) était dans l'obligation de pénétrer dans la propriété des époux de Sigalas, pour faire tous travaux d'études et de mensuration. A cette fin, elle obtint un arrêté du préfet de la Haute-Vienne en date du 22 août 1923 : tout ce qui est pénétration dans les propriétés privées pour les travaux d'études, de mensuration et de nivellement nécessaires à l'établissement des chutes concédées, était régi à ce moment par le décret du Président de la République du 20 janvier 1923 (2) ; il permet au demandeur en concession de procéder chez les tiers à tous travaux de nivellement, d'études et de mensuration, à la condition d'obtenir un arrêté préfectoral; le demandeur doit seulement exécuter toutes les prescriptions de l'article premier de la loi du 29 décembre 1892, bien connue dans le monde des entrepreneurs sous ce titre : "Loi de l'occupation temporaire ". Il nous suffira d'extraire de cet article premier les phrases suivantes, pour faire connaître les prescriptions auxquelles la Société concessionnaire était tenue de se conformer : "L'arrêté qui " autorise la pénétration, doit être affiché à la mairie de la " commune et présenté à toute réquisition.... La pénétration " des agents ne peut avoir lieu que cinq jours après la notification "de l'arrêté au propriétaire ou au gardien.... Il ne peut être " abattu d'arbres fruitiers, d'ornement ou de haute futaie avant " qu'un accord soit intervenu ou qu'il ait été procédé à une " constatation contradictoire destinée à fournir des éléments " nécessaires pour l'évaluation des dommages.... A la fin de "l'opération, tout dommage causé par les études est réglé entre "le propriétaire et l'Administration dans les formes indiquées "par la loi du 22 juillet 1889."

Si l'on se rappelle que cette dernière loi a organisé la procédure

(1) Cet arrêt du 22 juin 1928 a été publié au $8^{\mathrm{e}}$ cahier de Dalloz $1928,3^{\circ}$ partie, page 48 , avec les conclusions de $M$. Josse, Commissaire du Gouvernement et une note de M. Pépy.

(2) Voir le texte de ce décret au Bulletin de la Chambre Syndicale des Forces Hydrauliques, année 1923, No 396 ; il a été remplacé par un autre décret du 20 décembre 1926 (voir ibidem No 767, année 1927). devant les Conseils de Préfecture, on comprendra facilement que l'appréciation de tous les dommages occasionnés ou allégués en raison des travaux exécutés, pour études, nivellements, mensurations, soit attribuée à la juridiction administrative.

Il est non moins facile de comprendre ce qui est arrivé dans la suite : c'est l'événement classique ; les propriétaires prétendirent que les agents de la Société saccageaient leurs arbres et que l'abatage avail eu lieu sans constat préalable. Le 20 octobre 1923, ils faisaient défense à la Société de continuer ses opérations; le 21 novembre suivanf, ils l'assignaient en réparation devant le Conseil de Préfecture de la Haute-Vienne; le 26 décembre, le Conseil nommait des experts pour évaluer le dommage, et leur enjoignait de faire connaitre si la défense signifiée par les époux de Sigalas à la Société lui avait causé un préjudice en l'obligeant à suspendre ses travaux. Le rapport des experts conclut à 3.475 francs pour dommages aux époux de Sigalas résultant de l'abatage des arbres et à 100.000 francs pour dommages résultant pour la Société de la suspension de ses opérations. Le Conseil de Préfecture rendait le 14 avril 1925 un arrêté définitif allouant aux propriètaires 3.475 franes d'indemnité à raison des arbres abattus, mais les condamnant à 15.000 francs pour l'arrèt intempestif dont ils étaient la cause (avec deux tiers des dépens et les frais de l'expertise à leur charge). Un appel au Conseil d'Etat fut immédiatement interjeté par les époux de Sigalas.

Une première question de procédure se présentait devant le Conseil qui l'a écartée, puisqu'il n'en a même pas parlé : la Société avait-elle réellement fait une demande (principale ou reconventionnelle) pour obtenir des dommages-intérêts, dans cette instance où elle figurait comme défenderesse, ayant été assignée en paiement de dommages pour arbres abattus ? Et le motif du rejet est très simple : en supposant la procédure établie avec la plus parfaite régularité, la partie du procès relative à la demande de la Société, ne pouvait être jugée par le Conseil de Préfecture; opposer un obstacle à l'exécution d'une prescription administrative, même quand cet obstacle est méchamment et indûment organisé, ce n'est pas faire naître un débat qui rentre dans le cadre des travaux publics; il ne faut pas confondre un fait commis par une personne contre une autre (acte dont l'interprétation et les conséquences seront soumises à la Juridiction judiciaire) avec une question relative à l'empla- 
cement ou a l'entretien d'un ouvrnge " public " dont le contencieux est dévolu à la Juridiction administrative (1).

La solution donnée par le Conseil d'Etat entraîne la définition de la concession hydraulique. - Mais la question beaucoup plus grave était la suivante : le Conseil de Préfecture était-il compétent, mème pour juger le différend soulevé par les époux de Sigalas sur la question de leurs arbres, et du dommage dont ils se plaignaient ?

Les personnes peu renseignées sur la rigueur des principes administratifs, s'étonneront peut-ètre d'une pareille question : le décret du 20 janvier 1923 qui a permis au Préfet de prendre un arrèté d'autorisation, pour faciliter les mesures de nivellement et de mensuration, ne renvoie-t-il pas à la loi du 29 décembre 1892, laquelle prévoit expressément la compétence de la Juridiction administrative ? Comment donc discuter l'at tribution du litige à cette Juridiction ?

On ne saurait toutefois perdre de vue que si un décret peut statuer sur toutes les formalités à accomplir, il ne peut, sur la question de compétence, même en renvoyant à une loi, troubler l'ordre qui est établi par le principe de la séparation des pouvoirs : le Conseil de Préfecture ne peut commaître que des dommages causés par les travaux publics; d'où il ressort que la difficulté se synthétise de la façon suivante : la concession donnée en la forme prévue par la loi du 16 octobre 1919 est-elle une concession de travaux publics?

Si l'on consulte les travaux préparatoires, on voil que les rédacteurs de la loi nouvelle se sont toujours inspirés de la législation minière; on sait que pour statuer sur les conflits qui, en matière de mines, surgissent entre le concessionnaire et les propriétaires dont il occupe les terrains, le tribunal civil est compétent; or, d'après l'article 4 de la loi du 16 octobre, la mème juridiction connaitra des questions d'indemnité entre les propriétaires et le concessionnaire, et même de la fixation du prix d'acquisition si l'occupation doit dépasser la durée prévue au cahier des charges; et cette disposition rappelle étrangement l'article 43 de la loi du 21 avril 1810, modifié par la loi du 27 juillet 1880 , ainsi conçu : «Le concessionnaire peut être autorisé par arrêté préfectoral pris après que les propriétaires auronl, été " mis à même de présenter leurs observations, à occuper dans le "périmètre de sa concession, les terrains nécessaires à l'exploita-

(1) Il paraît que les obstacles élevés par les propriétaires à l'encontre des occupations temporaires, sont fréquents; mais la jurisprudence du Conseil d'Etat est constante : il ne veut point que ces obstacles soient jugés par la Conseil de Préfecture : on peut consulter l'arrêt du 26 février 1913 (Recueil du Conseil d'Etat, page 273, affaire GaHard) sur l'obstruction apportée par le propriétaire d'une carrière à l'utilisation de celle-ci par un entrepreneur chargé de l'empierrement d'une route. Et une demande reconventionnelle ne peut pas avoir pour effet de rendre compétente une juridiction qui, à raison même de la nature des débats, ne peut pas en connaître. Ainsi le Conseil d'Etat, statuant sur une faute de service commise par un agent de l'administration militaire, peut parfaitement statuer à la demande d'un particulier sur les conséquences de cette faute et admettre une responsabilité partagée entre l'agent de l'Etat et le particulier propriétaire d'une automobile qui a été endommagée (affaire Morel ; arrêt du Conseil d'Etat du 12 avril 1924 ; rendu au sujet d'une décision du ministre qui rejetait toute responsabilité de la part de l'Etat; Recueil du Conseil d'Etat. page 412). Mais, le Conseil d'Etat ne peut pas statuer sur le recours de l'Etat contre un particulier qui a endommagé une automobile appartenant à l'Etat : arrêt du 23 janvier 1924 (affaire Société La Providence, Recueil du Conseil d'Etat, page 80). En d'autres termes, on ne peut, par la voie de la "reconvention", demander à un tribunal de statuer sur un litige qui, par voie principale, ne saurait lui être soumis, à raison de son incompétence pour le juger.
" tion de sa mine, ...Si les travaux entrepris par le concessionnaire " ou par un explorateur, muni du permis de recherche ne sont que " passagers, et si le sol oû ils ont eu lieu, peut être mis en culture " au bout d'un an, comme il l'était auparavant, l'indemnité " sera réglée à une somme double du produit net du terrain " endommagé. Lorsque l'occupation ainsi faite prive le pro" priétaire de la jouissance du sol pendant plus d'une année, " ou lorsqu'après l'exécution des travaux les terrains occupés " ne sont plus propres à la culture, les propriétaires peuvent " exiger du concessionnaire ou de l'explorateur, l'acquisition a du sol.. Les contestations relatives aux indemnités réclamées " par les propriélaires du sol aux concessionnaires de mines, en " vertu du présent article, seront soumises aux Tribunaux civils."

On comprend dès lors, combien il était tentant pour les époux de Sigalas de soutenir l'incompétence du Tribunal administratif bien qu'ils l'eussent eux-mêmes saisi, mais ils croyaient que, pour se déclarer incompétente sur la question du paiement auquel ils avaient été personnellement condamnés, la juridiction administrative devait préalablement se déclarer incompétente sur la demande principale, c'est-à-dire sur leur propre demande.

L'arrêt dont on lira ci-dessous le texte a tranché la question de la façon suivante :

$1^{\circ}$ L'article 4 de la loi du 16 octobre 1910 qui attribue à la Juridiction civile la compétence nécessairc pour fixer les indemnités dues aux propriétaires dont les terrains seront grevés des servitudes énoncées à cet article, a un caractère exceptionnel : on ne doit pas le faire sortir du cadre pour lequel il a été strictement créé.

$2^{\circ}$ Mais, cette disposition exceptionnelle n'empeche pas que la concession hydraulique soit une concession de travaux publics, avec toutes les conséquences que comporte ce principe, dont la principale est la suivante : les dommages occasionnés par les ouvrages placés par le concessionnaire, seront déférés au Conseil de Préfecture.

Il nous reste à expliquer dans quels principes cette déciston a été puisée.

Considérations contenues dans l'arrêt du Conseil d'Etat. Le Conseil réunit en une seule phrase, différents caractères de la concession hydraulique donnée en vertu de la loi nouvelle.

$1^{\circ}$ La loi déclare que tous les ouvrages établis par le concessionnaire feront retour à l'Etat à l'expiration de la concession. En d'autres termes, les ouvrages dès qu'ils sont placés par le concessionnaire appartiennent à l'Etat qui en aura la jouissance quand la date prévue au cahier des charges sera arrivée.

$20 \mathrm{La}$ loi crée au profit et à la chárge du concessionnaire, un système de droits el obligations destinés à assurer, dans le présént et dans l'avenir, le meilleur emploi de la richesse naturelle des cours d'eau dont la puissance publique concède l'usage en vue de pourvoir aux besoins de la collectivité.

$3^{\circ} \mathrm{La}$ loi dispose que le concessionnaire devra constituer des réserves en force et en eau qui seront tenues par lui à la disposition des services publics, selon les conditions inscrites au cahier des charges pour leur utilisation.

$4^{\circ}$ Elle prévoit en outre que des tarifs maxima seront arrêtés tant pour lesdites réserves que pour les ventes d'énergie au public, s'il y a lieu.

Et l'arrêt ajoute : “ De l'ensemble de ces dispositions, il résulte " que les travaux du concessionnaire ont le caractere de travaux " publics, et que, par suite, les actions afférentes aux dommages " qu'ils peuvent occasionner, ne relèvent en principe que du "Conseil de Préfecture." 
Si toutes les concessions données par décret rentraient dans le cadre délini par ce que l'on vient de lire, en ce qui concerne la question des réserves et du tarif maximum, nous croyons que la décísion de la Haute Juridiction ne trouverait que des approbations. On peut même dire que ce texte s'applique parfaitement aux concessions relatives à certaines usines hydrauliques destinćes à alimenter le public en courant électrique, soit à fournir ce courant à des entreprises de distribution. Elles ressemblent alors à une concession bien antérieure à la loi clle-même, concession donnée par décret du 25 octobre 1914, avec déclaration d'utilité publique à la Société des Grands Travaux de Marseille et qui avait pour but unique de vendre de l'énergie au public (article 15) avec obligation stricte de fournir le courant qui lui scrait demandé (art. 17).

A plus forle raison, les caractères analysés par le Conseil d'Etat s'appliquent encore mieux à la concession obtenue par la Société Energie Electrique du Littoral Méditerranéen, concession dite de Sainte-Tulle accordée par le décret du 8 octobre 1920 (voir Bulletin de la Chambre Syndicale des Forces Hydrauliques, No 312,1920 ). Il est bien évident qu'une Société qui est dans toute une région, distributrice de courant électrique, ne le produit pas pour une autre raison que celle d'en faire la distribution. Ainsi que le remarque M. Josse dans ses conclusions, l'arrêt du Conseil d'Etat du 8 mai 1924 (qui a imposé à la patente des entrepreneurs de travaux publics la Société des Grands Travaux de Marseille pour la construction du barrage de Beaumont-Monteux) et celui du 5 août 1925 (qui a déclaré le Conseil de Préfeclure compétent pour statuer sur l'asséchement des puits de divers particuliers à la suite de la construction du barrage de Sainte-Tulle) se comprennent admirablement. (Voir Dalloz 1926.3.69.)

Mais la concession soumise au Conseil d'Etat, donnée à ủne société métallurgique était à peine relative à une fourniture de courant; nous trouvons en effet dans l'article premier du cahier des charges le texte suivant : " L'entreprise a pour objet prin" cipal . la fourniture de l'énergie à des établissements industriels " (électrochimie et électrométallurgie) exploités par le conces" sionnaire, principalement dans le département de la Haute"Vienne; et éventucllement, dans la limite des disponibilités " laissées par l'industrie sus-visée, la vente de l'énergie au pu" blic. " Le Commissaire du Gouvernement a très nettement aperçu la difficulté et la grande complication à laquelle il allait aboutir s'il demandait au Conseil d'Etat de considérer comme concessions de travaux publics, les seules concessions qui seraient données pour alimenter des services publics.

Et pour arriver à la simplification que donne toujours une parfaite unification, il se rattache à cette idée que l'article 21 du cahier des charges prévoit, dès l'origine, une réserve en force de 500 kilowatts au profit des services publics et des collectivités administratives. Mais, il est regrettable qu'il ne se soit pas mis résolument en présence de certaines concessions dans lesquelles on ne trouverait ni conditions imposées pour la vente au public, ni même une obligation de maintenị des réserves à la disposition des collectivités. Par exemple, dans le décret du 8 mars 1928 (Bulletin de la Chambre Syndicale des Forces hydraullques, avril 1928, No 885), on lit la concession d'une chute sur l'Ariège, au lieu dit de Luzenac, concédée à la Société des Talcs de Luzenac dans laquelle on chercherait vainement, même l'éventualité d'une distribution et, à plus forte raison, un tarif quelconque. L'article Ier du cahier des charges stipule senlement : "L'entreprise a pour objet principal l'alimentation en "énergie mécanique et électrique des établissements industriels " que le concessionnaire exploite dans le département de l'Arlège." tet l'on ne dira point que e'est une erreur de redaction, ptistque au chapitre IV intitulé dans le cahier des charges-type "Vente de l'énergie au public ", on lit une note ainsi conçue : Ce chapitre " ne devra pas figurer dans le cahier des charges, s'il est constant " que le concessionnaire ne fera pas de vente de l'énergie au " public pendant toute la durée de la concession."

Citons maintenant une concession donnée par décret du 17 août 1922 (voir Bulletin de la Chambre Syndicale des Forces Hydrauliques, année 1922, $\mathrm{N}^{\circ}$ 376); il s'agit de la concession donnée pour la régularisation du Doubs par la constitution d'une réserve d'eau dans les lacs de Saint-Point et de Rémoray ; nous avons sons les yeux le texte du cahier des charges et nous $y$ voyons une série d'articles dont la copie, ainsi qu'on va le vair, n'est pas compliquée; car si on lit dans l'article premier : « La "concession à laquelle"s'applique le présent cahier des charges " a pour objet l'établissement et l'exploitation des ouvrages " hydrauliques destinés à constituer dans les lacs de Saint-Point " et Rémoray, une réserve d'eau ulilisable de 14 millions de mètres " cubes pour régulariser le débit du Doubs et améliorer le régime "des usines d'aval ", on trouve dans les aulres articles une rédaction uniforme: "article 19 (tarif maximum) : néant. - Arti" cle 20 (obligation de fournir le courant) : néant. - Article 21 "(réserves en eau) : néant. - Article 22 (réserve au profit des " services publics : néant. - Article 23 (accords intervenus): " néant. - Article 24 (réserves d'énergie à laisser dans les dépar" tements riverains) : néant. - Article 25 (tarifs applicables aux " services publics) : néant. - Article 26 (tarifs applicables aux " réserves d'énergie à laisser dans les départements riverains) : " néant, "

Faudra-t-il, pour faire entrer dans le cadre des concessions de travaux publics, la concession du 17 abût 1922, muette sur les réserves et sur le tarif maximum, attendre que le cahier des charges soit modifié et qu'à la place du mot "néant "si souvent répété, on lise un tarif maximum ou une stipulation relalive à des fournitures aujourd'hui inexistantes, en faveur des services publics, ou même, simplement, du " public " ?

Nous ne le pensons pas, et nous croyons que l'orientation du Conseil d'Etat est si nette dans le sens d'une unification compliete, cqu'il jugerait, pour une concession en la forme de celle domice à la Société des lacs de Saint-Point et de Remoray, ce qu'il a jugé pour la concession domnée à la Société Electrométallurgíque du Palais.

Il lui suffira de retenir comme déterminants au point de vue de la compétence administrative, non point l'ensemble des quatre caractères que nous avons reproduits ci-dessus, en les puisant dans le texte même de l'arrèt, mais ces deux scu's èléments, savoir :

$1^{\circ}$ Dans toute concession donnée en la forme de la loi du 16 octobre 1919, il est spécifié que les ouvrages destinés à la production de l'énergịe et le bâtiment quị les abrite, font relour à l'Etat.

$2^{\circ}$ La mise en valeur de l'énergie que représente le cours de nos rivières, est faite " dans l'intérêt général ".

Avec la tendance qui pousse le Conseil d'Etat depuis quelque temps, à faire dépendre l'idée de "travail public " non pas de "l'utilité publique ", mais de "l'intérêt général " auquel c travail sera consacré, nous croyons que la réunion de ces deux c̀éments constituera pour lui une condition nécessaire mais suffisante, pour la thèse qqu'il veut généraliser.

Sans doute, à elle scule, la première condition " construction " par une Administration ou son concessionnaire d'un ouvrage "devant appartenir ou faire retour à celle Administration " ne surfirait pas à entenîner le classement dudit ouvrage dans le 
compartiment des travaux publics. Il arrive constamment que l'Etat ou une Ville exécutent ou font exécuter des travaux pour leur compte sans que ceux-ci se distinguent d'un travail qu'accomplirait un particulier. Aucun service public n'est intéressé à cette construction; aucun intérèt vraiment général n'y est attaché. C'est, à notre avis, pour cette considération, que le Conseil d'Etat, dans un arrèt très connu du 7 avril 1916 (affaire. Astruc; Recueil du Conseil d'Etat, page 164) n'a pas voulu confier au contentieux administratif, l'examen d'une convention signée par la ville de Paris pour la construction d'un théâtre aux Champs-Elysées, bien que l'édifice dut être remis à la ville à l'expiration d'un certain nombre d'années convenues; ce nouveau thêâtre ne pouvait évidemment pas rentrer dans un service public; d'autre part, il est difficile de dire, si attrayants que soient pour certaines personnes, les théâtres des ChampsElysées, qu'ils sont empreints d'un caractère d'intérèt général

Mais si ce "caractère d'intérêt général " peut être attribué à des ouvrages établis par une personne publique, il sera suffisant, d'après l'orientation de la jurisprudence actuelle, pour leur donner la consécration officielle de travaux publics et, par conséquent, en attribuer le contentieux aux Tribunaux administratifs. Il n'est plus nécessaire que l'on y trouve un caractère d'utilité publique, cette expression étant singulièrement plus étroịte que "l'intérêt général ".

L'utilité publique évoque en notre esprit l'idée d'un service public : en effet, si l'Administration crée un service dans l'idée de donner de grandes facilités au "public " (chemins de fer, distributions d'eau ou de gaz,... etc...), il est tout naturel de dire que "l'ouvrage qui fera partie intégrante de ce service, sera " d'utilité publique \%. L'intérêt général évoque, au contraire, dans notre esprit, une idée moins bien définie, mais essentiellement plus vaste. Ces mots s'appliquent à tout ouvrage susceptible d'être utilisé par une collectivité très étendue ou de faire sentir ses effets bienfaisants sur une foule de personnes, alors même qu'il nous serait impossible de dire quels sont les individus qui s'en serviront.

Les Offices publics d'habitations à bon màrché institués par la loi du 23 décembre 1912, et créés avec la personnalité morale ne constituent pas des " services publics", mais des sociétés qui, dans certaines conditions, s'administrent elles-mèmes ; mais ils ont pour objet exclusif, l'aménagement, la construction et la gestion d'immeubles salubres, ainsi que l'assainissement de maisons existantes et la création de cités jardins ou de jardins ouvriers. Ils ont done un but d'intérêt général; et le seul fait d'avoir passé un marché d'entreprise avec un Office de cette nature, ou avec l'entrepreneur choisi par lui, fait tomber sous le coup de la "patente des entrepreneurs de travaux publics" le sous-traitant qui a contracté ; c'est ce que nous enseigne l'arrêt du 30 décembre 1927 (affaire Tallet, Recueil Hebdomadaire de Dalloz, 1928, № 9, page 140) : un Office de cetle nature, nous dit cet arrêt, constitue un établissement public auquel sont assignées pour objet exclusif, la 'construction et la gestion d'immeubles salubres; les travaux que fait effectuer cet office (personne morale administrative) ont un but “ d'intérêt général " : ils sont des travaux publics. Il nous serait cependant impossible d'indiquer même d'une manière approximative quelles personnes en profitent ; elles appartiennent toutes à cetle grande collectivité des ouvriers et employés qui ne peuvent s'offrir un appartement luxueux.

Mais, l'arrêt le plus précieux à consulter est bien certainement celui du 10 juin 1921 rendu dans l'affaire des époux Lalanne et de l'enfant Brousse contre la commune de Monségur (Dalloz 1921.3.27) sur les remarquables conclusions de M. le Commis- saire du Gouvernement Corneille; les circonstances étaient les suivantes : postérieurement à la dernière des lois sur la séparation de l'Eglise et de l'Etat, en juin 1908, un groupe d'enfants pénètre dans l'église de Monségur' ; ils se suspendent, pour faire des exercices d'acrobatie, au bénitier qui, élevé sur une colonne, bascule et se brise en coupant la jambe de l'enfant Brousse. Il n'est plus possible de dire que, depuis la séparation, il y a un " service public du culte "; la commune est par l'article 5 de la loi du 2 janvier 1907, simplement propriétaire de l'église qui reste à la disposition de la communauté des fidèles. Cela suffit au Conseil d'Etat pour dire que "les travaux exécutés dans une " église pour le compte d'une personne publique dans un but " d'utilité générale, conservent le caractère de travaux publics, " et que les actions dirigées contre les communes à raison des " dommages provenant du défaut d'entretien des églises ren" trent dans la compétence du Conseil de Préfecture comme se "rattachant à l'exécution ou à l'inexécution d'un travail pu" blic. " Il retient la compétence de la juridiction administrative et, comme juge du second degré, il exonère la commune de toutes les condamnations prononcées contre elle, pour ce motif que les bénitiers ne sont,pas faits pour servir de trapèze aux enfants.

Comme il est toujours intéressant de connaître les tendances du Tribunal judiciaire à côté de celles du Tribunal administratif, on peut aussi se reporter à un arrêt de la Cour de Cassation du 4 mai 1926 (Dalloz 1927.1.126; affaire Mutin contre ville de Dinan et autres) dans lequel la Chambre civile a déclaré que si une commune propriétaire d'une église, est assignée pour le paiement des travaux de réparations à un orgue commandés par le curé avant la séparation de l'Eglise et de l'Etat, il appartient au Conseil de Préfecture seul de connaître de la difficulté.

Observations en ce qui concerne les travaux des mines. Si l'intérêt général qui s'attache à un ouvrage et ce fait que l'Etat doit devenir, à l'expiration d'un certain délai, propriétaire de cet ouvrage, suffisent pour faire rentrer ce dernier dans le cadre d'un travail public, que devra-t-on décider au sujet des "mines "? Personne n'est indifférent à la production du charbon; donc, l'intérêt général qui s'attache à une mine ne saurait se discuter; d'autre part, depuis la loi du 9 septembre 1919, les mines nouvellement concédées (et celles-là seulement, à l'exclusion des concessions antérieures) sont exploitées pendant 50 ou 99 ans, par le concessionnaire, et le retour gratuit à l'Etat en fin de concession, comporte non seulement le gisement minier, mais les bâtiments, ouvrages, machines, appareils et engins de toute nature compris dans les dépendances immobilières de la concession.

Si l'on fait dépendre de l'existence d'un tarif maximum et de l'obligation de constituer des réserves, le caractère de travail public d'une chute concédéc, la distinction entre la chute d'eau et la mine (qui ne connaît ni la sujétion à des tarifs, ni l'obligation à la constitution de réserves) est très facile : plus délicate, au contraire, sera-t-elle, si l'on définit comme nous venons de le faire, le caractère de travail public de la chute concédée, par " la simple coexistence de l'intérêt général et du retour à l'Etat. "

On peut toutefois remarquer certaines différences. Le cahier des charges d'une concession hydraulique spécifie nettement les travaux que devra effectuer le concessionnaire, et on sent que l'Etat intervient d'une manière permanente dans ces travaux, pour les énumérer (article 6 du cahier des charges), en approuver le projet (article 8), leur imposer des délais d'exécution et en faire la réception (article 9), en exiger le bornage (article 11). On ne trouve rien de semblable dans le domaine des mines, et il est évident que le concessionnaire minier travaille chez lui et pour lui, en supportant simplement la participation de l'Etat et du 
personnel aux bénćfices de la mine. Si l'abandon à la fin de la concession nouvelle s'impose, il faut y voir une satisfaction donnée par le législateur aux idées modernes, plus qu'un bouleversement dans la législation déjà si ancienne des mines. Et si le Conseil d'Etat a statué dernièrement entre le concessionnaire d'une mine et le Ministère des Travaux publics (arrêt du 9 novembre 1927 ; Compagnie des Charbonnages de Lyon, Revue des Concessions année 1928, page 143) au sujet de la redevance qui est due à l'Etat, le Commissaire du Gouvernement a eu soin de faire remarquer que le Conseil statuait directement, sans intervention du Conseil de Préfecture, simplement sur le conflit élevé entre le concessionnaire et le Ministre pour un compte litigieux au sujet "d'un traité passé par l'Etat ". C'est ainsi que le Commissaire du Gouvernement a désigné l'acte de concession.

Conséquences pratiques de la décision rendue. -- Si l'on admet que, d'une manière définitive, les concessions hydrauliques rentrent dans le domaine des concessions de travaux publics, il faudra retenir que les Tribunaux administratifs auront pleine compétence pour juger les difficultés qui se produiront entre l'Administration et son concessionnaire, conformément d'ailleurs à ce qui est écrit dans l'article 59 du cahier des charges qui devient inattaquable et est ainsi conçu : "Les contestations qui " s'élèveront entre le concessionnaire et l'Administration, au “ sujet de l'exécution et de l'interprétation du présent cahier des " charges, seront jugées par le Conseil de Préfecture du dépar" tement du siège de l'usine."

Mais des conflits pourront, entre le concessionnaire et des particuliers, être soumis à la Juridiction administrative. Il en sera ainsi en matière d'accidents (voir arrêt de la Cour de Cassation du 26 décembre 1927, affaire Buguet-Billard ; communication du contentieux de la Chambre Syndicale des Forces Hydrauliques, No 541, année 1928, page 121) à moins toutefois que la victime allègue une imprudence susceptible de constituer un délit de la part du concessionnaire (même communication, arrêt Casanova de la Chambre des Requêtes du 2 mai 1927).

Les dommages à la propriété seront justiciables du Conseil de Préfecture, notamment les asséchements des puits du voisinage de la dérivation (arrêt du Conseil d'Etat du 5 août 1925 ; Energie Electrique du Littoral Méditerranéen, Dalloz 1926.3.69). Les entrepreneurs de la construction seront soumis à la patente d'entrepreneurs de travaux publics (Conseil d'Etat, 8 mai 1924 ; Société des Grands Travaux de Marseille; Dalloz 1926.3.65).

L'enregistrement des concessions aurait été soumis au tarif des concessions de travaux publics, si la loi du 11 juillet 1928 n'avait pas ordonné l'application du seul tarif du droit fixe (22 fr. 50). Le texte de ladite loi a été reproduit dans le Recueil des Lois nouvelles, année 1928, No 14, page 629.

Arrêt du Conseil d'Etat du 22 juin 1928. - Vu la loi du 28 Pluviôse an 8. - Vu la loi du 29 déc. 1892. - Vu la loi du 16 oct. 1919. - Vu le décret du 20 janvier 1923.

Sur la compétence du Conseil de Préfecture pour connaître de la demande d'indemnité des époux de Sigalas : Considérant que les ouvrages établis par le concessionnaire d'une usine hydraulique sous le régime de la loi du 16 octobre 1919 doivent faire retour à l'Etat à l'expiration de la concession; que la loi crée au profit et à la charge du concessionnaire un système de droits et d'obligations destinés à assurer dans le présent et dans l'avenir le meilleur emploi de la richesse naturelle des cours d'eau dont la puissance publique concède l'usage en vue de pourvoir aux besoins de la collectivité ; que, notamment, la loi dispose que le concessionnaire devra constituer des réserves en force et en eau qui seront tenues par lui à la disposition des services publics selon les conditions inscrites au cahier des charges, et en vue desquelles il devra effectuer les travaux nécessaires pour leur utilisation ; qu'elle prévoit, en outre, que des tarifs maxima seront arrêtés tant pour lesdites réserves que pour les ventes d'énergie au public, s'il y a lieu.

Considérant que de l'ensemble de ces dispositions, il résulte que les travaux du concessionnaire ont le caractère de travaux publics et que, par suite, les actions afférentes aux dommages qu'ils peuvent occasionner ne relèvent, en principe, que du Conseil de Préfecture ;

Considérant, il est vrai, que l'article 4 prévoit que pour les servitudes qu'il envisage la compétence sera celle des tribunaux judiciaires; mais que cette compétence, toute exceptionnelle, doit être limitée strictement aux cas énumérés par ledit article; que, dans toutes les autres circonstances, les demandes d'indemnité pour dommages causés par les travaux du concessionnaire doivent ètre portées devant le Conseil de préfecture.

Considérant que les dommages causés par l'occupation de terrains pour travaux d'études préliminaires ne sont pas visés au dit article 4 ; que, dans ces conditions, le règlement d'administration publique du 20 janvier 1923, pris pour l'application de l'art. 28 de la loi du 16 octobre 1919, a pu légalement reconnaître la compétence du Conseil de Préfecture pour statuer sur les demandes d'indemnités formées à raison des opérations de nivellement et de mensuration qu'exécute le demandeur en concession, auquel le concessionnaire doit être assimilé, en vue de déterminer l'emplacement des ouvrages définitifs;

Considérant que, par arrêté du préfet de la Haute-Vienne du 22 août 1923 la Société Hydroélectrique du Palais a été autorisée à occuper la propriété des époux de Sigalas en vue de procéder aux études nécessaires pour délimiter et borner le périmètre des terrains qui devaient être achetés par elle, conformément à l'acte de concession; que ces opérations rentraient parmi celles prévues à l'article 28 de la loi du 16 octobre 1919 ; que, par suite, le Conseil de Préfecture était compétent pour connaître de la demande d'indemnité formée par les époux de Sigalas;

En ce qui concerne la condamnation prononcée contre les époux de Sigalas au profit de la Société du Palais. - Considérant que si la Société du Palais estimait que les époux de Sigalas avaient usé de procédés abusifs et vexatoires pour l'empècher d'occuper la propriété dont s'agit, et de jouir ainsi des droits que lui conférait l'arrêté préfectoral, c'est devant l'autorité judiciaire, seule compétente pour en connaître, qu'elle devait porter sa réclamation; qu'il y a lieu par suite d'annuler l'arrêté attaqué en tant qu'il a condamné les époux de Sigalas à payer une indemnité à la Société du Palais et de mettre la totalité des dépens, y compris les frais d'expertise à la charge de ladite Socièté :

Art. 1 ${ }^{\mathrm{er}}$. - Les époux de Sigalas sont déchargés des condamnations prononcées contre eux par l'arrêté du Conseil de Préfecture de la Haute-Vienne en date du 14 avril 1925;

ART. 2. - Les dépens de première instance, y compris les frais d'expertise, sont mis à la charge de la Société du Palais ;

Arr. 3. - L'arrêté sus-visé du Conseil de Préfecture du département de la Haute-Vienne du 14 avril 1925 est réformé en ce qu'il a de contraire à la présente décision;

Art. 4. - Le surplus des conclusions de la requête des époux de Sigalas est rejeté.

MM. Seligman, rapp. ; Josse, concl. ; de Lavergne et BoivinChampeaux, av. 